\title{
Classes of harmonic starlike functions defined by Sălăgean-type $q$-differential operators
}

\author{
Jay Jahangiri*1 (D), Gangadharan Murugusundaramoorthy $^{2}$ (D), Kaliappan Vijaya ${ }^{2}$ (iD \\ ${ }^{1}$ Mathematical Sciences, Kent State University, Kent, Ohio, U.S.A. \\ ${ }^{2}$ Department of Mathematics,School of Advanced Sciences, Vellore Institute of Technology, Vellore - \\ 632014,T.N., INDIA
}

\begin{abstract}
Sufficient and necessary coefficient bounds, extreme points of closed convex hulls, and distortion theorems are determined for a family of harmonic starlike functions of complex order involving Sălăgean-type $q$-differential operators.
\end{abstract}

Mathematics Subject Classification (2010). 30C45, 30C50.

Keywords. harmonic univalent functions, $q$-calculus, Sălăgean-type differential operators.

\section{Introduction}

Let $\mathcal{A}$ denote the class of functions $h$ of the form

$$
h(z)=z+\sum_{n=2}^{\infty} a_{n} z^{n}
$$

which are analytic in the open unit disk $\mathbb{U}=\{z \in \mathbb{C}:|z|<1\}$. Also let $\mathcal{S}$ denote the subclass of $\mathcal{A}$ consisting of functions that are univalent in $\mathbb{U}$.

We now recall the notion of q-operators or q-difference operators that play vital roles in the theory of hypergeometric series, quantum physics and operator theory. The application of q-calculus was initiated by Jackson [7] who have used the fractional q-calculus operators in investigations of certain classes of functions which are analytic in $\mathbb{U}$. For more details on $q$-calculus and its applications one can refer to $[1,5,7,13]$ and the references cited therein.

For $0<q<1$ the Jackson's $q$-derivative of a function $h \in \mathcal{S}$ is given as follows [7]

$$
\begin{aligned}
D_{q} h(z)= & \begin{cases}\frac{h(z)-h(q z)}{(1-q) z} & \text { for } z \neq 0, \\
h^{\prime}(0) & \text { for } z=0,\end{cases} \\
& D_{q}^{2} h(z)=D_{q}\left(D_{q} h(z)\right) .
\end{aligned}
$$

From (1.2), we have $D_{q} h(z)=1+\sum_{n=2}^{\infty}[n]_{q} a_{n} z^{n-1}$ where $[n]_{q}=\frac{1-q^{n}}{1-q}$ is sometimes called the basic number $n$. If $q \rightarrow 1^{-}$then $[n]_{q}=[n] \rightarrow n$. For $h \in \mathcal{A}, m \in \mathbb{N}_{0}=\{0,1,2, \ldots\}$

\footnotetext{
* Corresponding Author.

Email addresses: jjahangi@kent.edu (J. Jahangiri), gmsmoorthy@yahoo.com (G. Murugusundaramoorthy), kvijaya@vit.ac.in (K. Vijaya)

Received: 05.07.2018; Accepted: 21.12.2018
} 
and $z \in \mathbb{U}$, Govindaraj and Sivasubramanian [5] considered the Sălăgean $q$-differential operators

$$
\begin{aligned}
D_{q}^{0} h(z) & =h(z) \\
D_{q}^{1} h(z) & =z D_{q} h(z), \ldots, \\
D_{q}^{m} h(z) & =z D_{q}\left(D_{q}^{m-1} h(z)\right)=z+\sum_{n=2}^{\infty}[n]_{q}^{m} a_{n} z^{n} .
\end{aligned}
$$

We note that if $q \rightarrow 1^{-}$then

$$
D^{m} h(z)=z+\sum_{n=2}^{\infty}[n]^{m} a_{n} z^{n} \quad\left(m \in \mathbb{N}_{0}, z \in \mathbb{U}\right)
$$

is the familiar Sălăgean derivative[15].

Let $\mathcal{H}$ denote the family of harmonic functions $f=h+\bar{g}$ that are orientation preserving and univalent in $\mathbb{U}$ with $h$ as in (1.1) and $g$ given by

$$
g(z)=\sum_{n=1}^{\infty} b_{n} z^{n}, \quad\left|b_{1}\right|<1
$$

We note that the family $\mathcal{H}$ of orientation preserving, normalized harmonic univalent functions reduces to the well known class $\mathcal{S}$ of normalized univalent functions if the co-analytic part of $f$ is identically zero, i.e. $g \equiv 0$. We let $\overline{\mathcal{H}}$ be the subfamily of $\mathcal{H}$ consisting of harmonic functions $f=h+\bar{g}$ for which $h$ and $g$ are given by

$$
h(z)=z-\sum_{n=2}^{\infty} a_{n} z^{n}, g(z)=\sum_{n=1}^{\infty} b_{n} z^{n}, a_{n} \geq 0 \text { and } b_{n} \geq 0 .
$$

The seminal work of Clunie and Sheil-Small [4] on harmonic mappings prompted many research articles on classes of complex-valued harmonic univalent functions. In particular, $[2,6,8,9,11,12,14,16]$ have investigated properties of various subclasses of harmonic univalent functions.

For harmonic functions $f=h+\bar{g} \in \mathcal{H}$ where $h$ and $g$ are, respectively, given by (1.1) and (1.4), let $D_{q}^{m} h(z)$ be defined by (1.3) and $D_{q}^{m} g(z)$ be defined by

$$
\begin{aligned}
D_{q}^{0} g(z) & =g(z) \\
D_{q}^{1} g(z) & =z D_{q} g(z), \ldots, \\
D_{q}^{m} g(z) & =z D_{q}\left(D_{q}^{m-1} g(z)\right)=z+\sum_{n=2}^{\infty}[n]_{q}^{m} b_{n} z^{n} .
\end{aligned}
$$

Recently, Jahangiri [10] considered a generalized Sălăgean $q$ - differential operator $\mathcal{H}_{q}^{m}(\alpha)$ defined by

$$
\Re\left(\frac{D_{q}^{m+1} f(z)}{D_{q}^{m} f(z)}\right) \geq \alpha ; 0 \leq \alpha<1,
$$

where, $D_{q}^{m} h(z)$ and $D_{q}^{m} g(z)$ are, respectively, defined by (1.3) and (1.5) and

$$
D_{q}^{m} f(z)=D_{q}^{m} h(z)+(-1)^{m} \overline{D_{q}^{m} g(z)}, m>-1 .
$$

The subfamily $\overline{\mathcal{H}}_{q}^{m}(\alpha) \subset \mathcal{H}_{q}^{m}(\alpha)$ consists of harmonic functions $f_{m}=h+\bar{g}_{m}$ for which

$$
h(z)=z-\sum_{n=2}^{\infty} a_{n} z^{n}, g_{m}(z)=(-1)^{m} \sum_{n=1}^{\infty} b_{n} z^{n}, a_{n} \geq 0 \text { and } b_{n} \geq 0 .
$$


For non-zero complex number $b$ with $|b| \leq 1$, real number $\gamma$ and $0 \leq \alpha<1$ we let $\mathcal{H} \mathcal{S}_{q}^{m}(b, \gamma, \alpha)$ be the subclass of $\mathcal{H}$ consisting of harmonic functions $f=h+\bar{g}$ satisfying

$$
\Re\left(1+\frac{1}{b}\left(\left(1+e^{i \gamma}\right) \frac{D_{q}^{m+1} f(z)}{D_{q}^{m} f(z)}-e^{i \gamma}-1\right)\right)>\alpha .
$$

We also let $\overline{\mathcal{H}} \mathcal{S}_{q}^{m}(b, \gamma, \alpha) \equiv \mathcal{H} \mathcal{S}_{q}^{m}(b, \gamma, \alpha) \cap \overline{\mathcal{H}}$.

We note that $\mathcal{H S}_{q}^{m}(1, \gamma, \alpha) \equiv \mathcal{H} \mathcal{R}_{q}^{m}(\gamma, \alpha)$ is generalized class of Goodman-Ronning-type harmonic starlike functions (see [14], Inequality (2), p. 46) satisfying

$$
\Re\left(\left(1+e^{i \gamma}\right) \frac{D_{q}^{m+1} f(z)}{D_{q}^{m} f(z)}-e^{i \gamma}\right)>\alpha
$$

and $\mathcal{H} \mathcal{S}_{q}^{m}(b, 0, \alpha) \equiv \mathcal{H} \mathcal{R}_{q}^{m}(b, \alpha)$ is the harmonic version of generalized starlike functions of complex order (see [3], Definition 1) satisfying

$$
\Re\left(1+\frac{2}{b}\left(\frac{D_{q}^{m+1} f(z)}{D_{q}^{m} f(z)}-1\right)\right)>\alpha .
$$

It is the aim of this paper to obtain sufficient coefficient conditions, extreme points, growth theorem, and distortion bounds for harmonic functions $f=h+\bar{g}$ in $\mathcal{H}_{q}^{m}(b, \gamma, \alpha)$. Moreover, we show that those sufficient coefficient conditions for $f \in \mathcal{H}_{q}^{m}(b, \gamma, \alpha)$ are also necessary for $f \in \overline{\mathcal{H}} S_{q}^{m}(b, \gamma, \alpha)$.

\section{Main results}

The sufficient coefficient condition for $\mathcal{H}_{q}^{m}(b, \gamma, \alpha)$ is given in the following theorem.

Theorem 2.1. Let $f=h+\bar{g} \in \mathcal{H}$ where $b$ is a non-zero complex number with $|b| \leq 1, \gamma$ is a real number and $0 \leq \alpha<1$. If

$$
\sum_{n=1}^{\infty}\left(\frac{[n]_{q}^{m}\left[2[n]_{q}-2+(1-\alpha)|b|\right]}{(1-\alpha)|b|}\left|a_{n}\right|+\frac{[n]_{q}^{m}\left[2[n]_{q}+2-(1-\alpha)|b|\right]}{(1-\alpha)|b|}\left|b_{n}\right|\right) \leq 2,
$$

then $f$ is harmonic univalent and orientation-preserving in $\mathbb{U}$ and $f \in \mathcal{H S}_{q}^{m}(b, \gamma, \alpha)$.

Proof. First we establish that $f$ is orientation preserving in $\mathbb{U}$. In other words, we need to show that $\left|D_{q}^{m+1} h(z)\right| \geq\left|D_{q}^{m+1} g(z)\right|$. This is accomplished using the properties of absolute values and the coefficient inequality (2.1).

$$
\begin{aligned}
\left|D_{q}^{m+1} h(z)\right| & \geq 1-\sum_{n=2}^{\infty}[n]_{q}^{m+1}\left|a_{n}\right| r^{n-1}>1-\sum_{n=2}^{\infty}[n]_{q}^{m+1}\left|a_{n}\right| \\
& \geq 1-\sum_{n=2}^{\infty}\left[\frac{2[n]_{q}-2+(1-\alpha)|b|}{(1-\alpha)|b|}\right][n]_{q}^{m}\left|a_{n}\right| \\
& \geq \sum_{n=1}^{\infty}\left[\frac{2[n]_{q}+2-(1-\alpha)|b|}{(1-\alpha)|b|}\right][n]_{q}^{m}\left|b_{n}\right| \\
& \geq \sum_{n=1}^{\infty}[n]_{q}^{m+1}\left|b_{n}\right| \geq \sum_{n=1}^{\infty}[n]_{q}^{m+1}\left|b_{n}\right| r^{n-1} \geq\left|D_{q}^{m+1} g(z)\right| .
\end{aligned}
$$

To show $f$ is univalent in $\mathbb{U}$ we use a method that was first used by Jahangiri [8]. We will show that $f\left(z_{1}\right) \neq f\left(z_{2}\right)$ when $z_{1} \neq z_{2}$. Consider $z_{1}$ and $z_{2}$ in $\mathbb{U}$ so that $z_{1} \neq z_{2}$. Since the unit disc $\mathbb{U}$ is simply connected and convex, we have $z(t)=(1-t) z_{1}+t z_{2}$ in $\mathbb{U}$ for $0 \leq t \leq 1$. Then we may write

$$
D_{q}^{m+1} f\left(z_{2}\right)-D_{q}^{m+1} f\left(z_{1}\right)=\int_{0}^{1}\left[\left(z_{2}-z_{1}\right)\left(D_{q}^{m+1} h(z(t))+\overline{\left(z_{2}-z_{1}\right)\left(D_{q}^{m+1} g(z(t))\right.}\right] d t .\right.
$$


Dividing the above equation by $z_{2}-z_{1}$ and taking the real parts we obtain

$$
\begin{aligned}
\Re\left(\frac{D_{q}^{m+1} f\left(z_{2}\right)-D_{q}^{m+1} f\left(z_{1}\right)}{z_{2}-z_{1}}\right) & =\int_{0}^{1} \Re\left[D_{q}^{m+1} h(z(t))+\frac{\overline{\left(z_{2}-z_{1}\right)}}{z_{2}-z_{1}} \overline{D_{q}^{m+1} g(z(t))}\right] d t \\
& >\int_{0}^{1}\left[\Re \left(D_{q}^{m+1} h(z(t))-\mid D_{q}^{m+1} g(z(t) \mid] d t .\right.\right.
\end{aligned}
$$

On the other hand

$$
\begin{aligned}
\Re\left(D_{q}^{m+1} h(z(t))-\mid\left(D_{q}^{m+1} g(z(t)) \mid\right.\right. & \geq \Re\left(D_{q}^{m+1} h(z(t))-\sum_{n=1}^{\infty}[n]_{q}^{m+1}\left|b_{n}\right|\right. \\
& \geq 1-\sum_{n=2}^{\infty}[n]_{q}^{m+1}\left|a_{n}\right|-\sum_{n=1}^{\infty}[n]_{q}^{m+1}\left|b_{n}\right| \\
& \geq 1-\sum_{n=2}^{\infty}[n]_{q}^{m}\left[\frac{2[n]_{q}-2+(1-\alpha)|b|}{(1-\alpha)|b|}\right]\left|a_{n}\right| \\
& -\sum_{n=1}^{\infty}[n]_{q}^{m}\left[\frac{2[n]_{q}+2-(1-\alpha)|b|}{(1-\alpha)|b|}\right]\left|b_{n}\right| \\
& \geq 0 \text { by }(2.1) .
\end{aligned}
$$

This together with inequality (2.2) implies the univalence of $f$.

Next we show that if the condition (2.1) holds then $f \in \mathcal{H S}_{q}^{m}(b, \gamma, \alpha)$. In other words, we need to show that the condition (1.7) is satisfied if (2.1) holds.

Using the fact that $\Re(w(z)) \geq \alpha$ if and only if $|1-\alpha+w| \geq|1+\alpha-w|$ for $0 \leq \alpha<1$ it suffices to show that

$$
\begin{aligned}
& \left|\left(2 b-\alpha b-e^{i \gamma}-1\right)\left(\mathcal{D}_{q}^{m} h(z)+(-1)^{m} \overline{\mathcal{D}_{q}^{m} g(z)}\right)+\left(1+e^{i \gamma}\right)\left(\mathcal{D}_{q}^{m+1} h(z)-(-1)^{m} \overline{\mathcal{D}_{q}^{m+1} g(z)}\right)\right| \\
& -\left|\left(1+\alpha b+e^{i \gamma}\right)\left(\mathcal{D}_{q}^{m} h(z)+(-1)^{m} \overline{\mathcal{D}_{q}^{m} g(z)}\right)\right|-\left(1+e^{i \gamma}\right)\left(\mathcal{D}_{q}^{m+1} h(z)-(-1)^{m} \overline{\mathcal{D}_{q}^{m+1} g(z)}\right) \mid \geq 0 .
\end{aligned}
$$

Upon substituting for $\mathcal{D}_{q}^{m} h(z)$ and $\mathcal{D}_{q}^{m} g(z)$ we obtain

$$
\begin{aligned}
& \mid\left(2 b-\alpha b-\left(1+e^{i \gamma}\right)\right)\left[z+\sum_{n=2}^{\infty}[n]_{q}^{m} a_{n} z^{n}+(-1)^{m} \sum_{n=1}^{\infty}[n]_{q}^{m} \overline{b_{n} z^{n}}\right] \\
& +\left(1+e^{i \gamma}\right)\left[z+\sum_{n=2}^{\infty}[n]_{q}^{m+1} a_{n} z^{n}-(-1)^{m} \sum_{n=1}^{\infty}[n]_{q}^{m+1} \overline{b_{n} z^{n}}\right] \mid \\
& -\mid\left(1+\alpha b+e^{i \gamma}\right)\left[z+\sum_{n=2}^{\infty}[n]_{q}^{m} a_{n} z^{n}+(-1)^{m} \sum_{n=1}^{\infty}[n]_{q}^{m} \overline{b_{n} z^{n}}\right] \\
& -\left(1+e^{i \gamma}\right)\left[z+\sum_{n=2}^{\infty}[n]_{q}^{m+1} a_{n} z^{n}-(-1)^{m} \sum_{n=1}^{\infty}[n]_{q}^{m+1} \overline{b_{n} z^{n}}\right] \mid
\end{aligned}
$$




$$
\begin{aligned}
\geq & (2-\alpha)|b||z|-\sum_{n=2}^{\infty}\left|(2-\alpha) b+\left(1+e^{i \gamma}\right)\left([n]_{q}-1\right)\right|[n]_{q}^{m}\left|a_{n}\right||z|^{n} \\
& -\sum_{n=1}^{\infty}\left|\left(1+e^{i \gamma}\right)\left([n]_{q}+1\right)-(2-\alpha) b\right|[n]_{q}^{m}\left|b_{n}\right||z|^{n} \\
& -\alpha|b||z|-\sum_{n=2}^{\infty}\left|\left([n]_{q}-1\right)\left(1+e^{i \gamma}\right)-\alpha b\right|[n]_{q}^{m}\left|a_{n}\right||z|^{n} \\
& -\sum_{n=1}^{\infty}\left|\left([n]_{q}+1\right)\left(1+e^{i \gamma}\right)+\alpha b\right|[n]_{q}^{m}\left|b_{n}\right||z|^{n} \\
\geq & 2(1-\alpha)|b||z|\left(1-\sum_{n=2}^{\infty}[n]_{q}^{m}\left[\frac{2\left[2[n]_{q}-2+(1-\alpha)|b|\right]}{2(1-\alpha)|b|}\left|a_{n}\right|\right]\right) \\
- & 2(1-\alpha)|b||z| \sum_{n=1}^{\infty}[n]_{q}^{m}\left[\frac{2\left[2[n]_{q}+2-(1-\alpha)|b|\right]}{2(1-\alpha)|b|}\left|b_{n}\right|\right] \\
\geq & 0, \text { by }(2.1) .
\end{aligned}
$$

The functions

$$
f(z)=z+\sum_{n=2}^{\infty}\left[\frac{(1-\alpha)|b|}{2[n]_{q}-2+(1-\alpha)|b|}\right] x_{n} z^{n}+\sum_{n=1}^{\infty}\left[\frac{(1-\alpha)|b|}{2[n]_{q}+2-(1-\alpha)|b|}\right] \bar{y}_{n} \bar{z}^{n}
$$

where $\sum_{n=2}^{\infty}\left|x_{n}\right|+\sum_{n=1}^{\infty}\left|y_{n}\right|=1$, shows that the coefficient bound given by (2.1) is sharp.

The next theorem shows that condition (2.1) is also necessary for $f \in \overline{\mathcal{H}} \delta_{q}^{m}(b, \gamma, \alpha)$.

Theorem 2.2. Let $f_{m}=h+\bar{g}_{m}$ be given by (1.6) where $b$ is a non-zero complex number with $|b| \leq 1, \gamma$ is a real number and $0 \leq \alpha<1$. Then $f_{m}$ is harmonic univalent and orientation-preserving in $\mathbb{U}$ and $f_{m} \in \overline{\mathcal{H}} S_{q}^{m}(b, \gamma, \alpha)$ if and only if

$$
\sum_{n=1}^{\infty}\left(\frac{[n]_{q}^{m}\left[2[n]_{q}-2+(1-\alpha)|b|\right]}{(1-\alpha)|b|} a_{n}+\frac{[n]_{q}^{m}\left[2[n]_{q}+2-(1-\alpha)|b|\right]}{(1-\alpha)|b|} b_{n}\right) \leq 2 .
$$

Proof. Since $\overline{\mathcal{H}} \delta_{q}^{m}(b, \gamma, \alpha) \subset \mathcal{H} S_{q}^{m}(b, \gamma, \alpha)$, the if part of the Theorem 2.2 follows from Theorem 2.1. To prove the only if part, we will show that if (2.3) does not hold then $f_{m}$ is not in $\overline{\mathcal{H}} \mathcal{S}_{q}^{m}(b, \gamma, \alpha)$.

For $f_{m} \in \overline{\mathcal{H}} \mathcal{S}_{q}^{m}(b, \gamma, \alpha)$ we must have

$$
\Re\left(1+\frac{1}{b}\left(\left(1+e^{i \gamma}\right) \frac{D_{q}^{m+1} h(z)-(-1)^{m} \overline{D_{q}^{m+1} g_{m}(z)}}{D_{q}^{m} h(z)+(-1)^{m} \overline{D_{q}^{m} g_{m}(z)}}-\left(e^{i \gamma}+1\right)\right)\right) \geq \alpha
$$


Or equivalently

$$
\begin{aligned}
& \Re\left(\frac{(1-\alpha) b z-\sum_{n=2}^{\infty}\left[(1-\alpha) b+\left([n]_{q}-1\right)\left(1+e^{i \gamma}\right)\right][n]_{q}^{m}\left|a_{n}\right| z^{n}}{b\left(z-\sum_{n=2}^{\infty}[n]_{q}^{m}\left|a_{n}\right| z^{n}+(-1)^{2 m} \sum_{n=1}^{\infty}[n]_{q}^{m}\left|b_{n}\right| \bar{z}^{n}\right)}\right) \\
& -\Re\left(\frac{(-1)^{2 m} \sum_{n=1}^{\infty}\left[\left([n]_{q}+1\right)\left(1+e^{i \gamma}\right)-(1-\alpha) b\right][n]_{q}^{m}\left|b_{n}\right| \bar{z}^{n}}{b\left(z-\sum_{n=2}^{\infty}[n]_{q}^{m}\left|a_{n}\right| z^{n}+(-1)^{2 m} \sum_{n=1}^{\infty}[n]_{q}^{m}\left|b_{n}\right| \bar{z}^{n}\right)}\right) \\
& =\Re\left(\frac{(1-\alpha)|b|^{2}-\sum_{n=2}^{\infty}\left[(1-\alpha) b+\left([n]_{q}-1\right)\left(1+e^{i \gamma}\right)\right] \bar{b}[n]_{q}^{m}\left|a_{n}\right| z^{n-1}}{|b|^{2}\left(1-\sum_{n=2}^{\infty}[n]_{q}^{m}\left|a_{n}\right| z^{n-1}+\frac{\bar{z}}{z} \sum_{n=1}^{\infty}[n]_{q}^{m}\left|b_{n}\right| \bar{z}^{[n]_{q}-1}\right)}\right) \\
& -\Re\left(\frac{\left.\frac{\bar{z}}{z} \sum_{n=1}^{\infty}\left[\left([n]_{q}+1\right)\left(1+e^{i \gamma}\right)-(1-\alpha) b\right] \bar{b}[n]_{q}^{m}\left|b_{n}\right| \bar{z}^{n-1}\right)}{|b|^{2}\left(1-\sum_{n=2}^{\infty}[n]_{q}^{m}\left|a_{n}\right| z^{n-1}+\frac{\bar{z}}{z} \sum_{n=1}^{\infty}[n]_{q}^{m}\left|b_{n}\right| \bar{z}^{n-1}\right)}\right) \geq 0 .
\end{aligned}
$$

The above condition must hold for all values of $\gamma,|z|=r<1$ and $0<|b|<1$. For $\gamma=0$ and $|b|=b$ let $z=r<1$ be on the positive real axis. Then the above condition becomes

$$
\begin{gathered}
\frac{(1-\alpha)|b|^{2}-\sum_{n=2}^{\infty}\left[\left(2[n]_{q}-2\right)+(1-\alpha) b\right]|b|[n]_{q}^{m}\left|a_{n}\right| r^{n-1}}{|b|^{2}\left(1-\sum_{n=2}^{\infty}[n]_{q}^{m}\left|a_{n}\right| r^{n-1}+\sum_{n=1}^{\infty}[n]_{q}^{m}\left|b_{n}\right| r^{n-1}\right)} \\
-\frac{\sum_{n=1}^{\infty}\left[\left(2[n]_{q}+2\right)-(1-\alpha) b\right]|b|[n]_{q}^{m}\left|b_{n}\right| r^{n-1}}{|b|^{2}\left(1-\sum_{n=2}^{\infty}[n]_{q}^{m}\left|a_{n}\right| r^{n-1}+\sum_{n=1}^{\infty}[n]_{q}^{m}\left|b_{n}\right| r^{n-1}\right)} \geq 0 .
\end{gathered}
$$

Now we observe that the numerator in the above required inequality (2.4) is negative if condition (2.3) does not hold. Thus, there exists a point $z_{0}=r_{0}$ in $(0,1)$ for which the quotient in the above inequalities are negative. This contradicts the required condition (1.7) for $f_{m} \in \overline{\mathcal{H}} S_{q}^{m}(b, \gamma, \alpha)$. Hence the proof is complete.

The following theorem is a consequence of the above Theorem 2.2.

Theorem 2.3. Let $f_{m}=h+\bar{g}_{m}$ be given by (1.6). Then $f_{m} \in \overline{\mathcal{H}} \delta_{q}^{m}(\gamma, \alpha)$ if and only if

$$
\sum_{n=1}^{\infty}\left(\frac{\left.[n]_{q}^{m}\left[2[n]_{q}-1-\alpha\right)\right]}{1-\alpha} a_{n}+\frac{\left.[n]_{q}^{m}\left[2[n]_{q}+1+\alpha\right)\right]}{1-\alpha} b_{n}\right) \leq 2 .
$$

The extreme points of closed convex hull of $\overline{\mathcal{H}} \delta_{q}^{m}(b, \gamma, \alpha)$, denoted by $c l c o \overline{\mathcal{H}} \delta_{q}^{m}(b, \gamma, \alpha)$, are determined in the following theorem.

Theorem 2.4. Let $f_{m} \in \operatorname{clco} \overline{\mathcal{H}} \delta_{q}^{m}(b, \gamma, \alpha)$ if and only if

$$
f_{m}(z)=\sum_{n=1}^{\infty}\left(X_{n} h_{n}+Y_{n} g_{m_{n}}\right)
$$

where

$$
h_{1}(z)=z, h_{n}(z)=z-\frac{(1-\alpha)|b|}{[n]_{q}^{m}\left[2[n]_{q}-2+(1-\alpha)|b|\right]} z^{n}, \quad n=2,3, \ldots
$$




$$
g_{m_{n}}(z)=z+(-1)^{m} \frac{(1-\alpha)|b|}{[n]_{q}^{m}\left[2[n]_{q}+2-(1-\alpha)|b|\right]} \bar{z}^{n}, \quad n=1,2, \ldots ;
$$

$\sum_{n=1}^{\infty}\left(X_{n}+Y_{n}\right)=1, X_{n} \geq 0$ and $Y_{n} \geq 0$.

In particular, the extreme points of clco $\overline{\mathcal{H}} \mathcal{S}_{q}^{m}(b, \gamma, \alpha)$ are $\left\{h_{n}\right\}$ and $\left\{g_{m_{n}}\right\}$.

Proof. For functions of the form (2.5), we have

$$
\begin{aligned}
f_{m}(z) & =\sum_{n=1}^{\infty}\left(X_{n} h_{n}+Y_{n} g_{m_{n}}\right) \\
& =\sum_{n=1}^{\infty}\left(X_{n}+Y_{n}\right) z-\sum_{n=2}^{\infty} \frac{(1-\alpha)|b|}{[n]_{q}^{m}\left[2[n]_{q}-2+(1-\alpha)|b|\right]} X_{n} z^{n} \\
+(-1)^{m} & \sum_{n=1}^{\infty} \frac{(1-\alpha)|b|}{[n]_{q}^{m}\left[2[n]_{q}+2-(1-\alpha)|b|\right]} Y_{n} \bar{z}^{n} .
\end{aligned}
$$

Therefore

$$
\begin{aligned}
& \sum_{n=2}^{\infty} \frac{[n]_{q}^{m}\left[2[n]_{q}-2+(1-\alpha)|b|\right]}{(1-\alpha)|b|}\left(\frac{(1-\alpha)|b|}{[n]_{q}^{m}\left[2[n]_{q}-2+(1-\alpha)|b|\right]}\right) X_{n} \\
& \quad+\sum_{n=1}^{\infty} \frac{[n]_{q}^{m}\left[2[n]_{q}+2-(1-\alpha)|b|\right]}{(1-\alpha)|b|}\left(\frac{(1-\alpha)|b|}{[n]_{q}^{m}\left[2[n]_{q}+2-(1-\alpha)|b|\right]}\right) Y_{n} \\
& =\sum_{n=2}^{\infty} X_{n}+\sum_{n=1}^{\infty} Y_{n}=1-X_{1} \leq 1 .
\end{aligned}
$$

Thus, $f_{m} \in \operatorname{clco} \overline{\mathcal{H}} S_{q}^{m}(b, \gamma, \alpha)$. Conversely, suppose that $f_{m} \in c l c o \overline{\mathcal{H}} \delta_{q}^{m}(b, \gamma, \alpha)$. Set

$$
X_{n}=\frac{[n]_{q}^{m}\left[2[n]_{q}-2+(1-\alpha)|b|\right]}{(1-\alpha)|b|}\left|a_{n}\right|, n=2,3, \ldots,
$$

and

$$
Y_{n}=\frac{[n]_{q}^{m}\left[2[n]_{q}+2-(1-\alpha)|b|\right]}{(1-\alpha)|b|}\left|b_{n}\right|, n=1,2, \ldots,
$$

where $\sum_{n=1}^{\infty}\left(X_{n}+Y_{n}\right)=1$. Then

$$
\begin{aligned}
f_{m}(z) & =z-\sum_{n=2}^{\infty} a_{n} z^{n}+(-1)^{m} \sum_{n=1}^{\infty} b_{n} \bar{z}^{n} \\
& =z-\sum_{n=2}^{\infty} \frac{(1-\alpha)|b|}{[n]_{q}^{m}\left[2[n]_{q}-2+(1-\alpha)|b|\right]} X_{n} z^{n}+(-1)^{m} \sum_{n=1}^{\infty} \frac{(1-\alpha)|b|}{[n]_{q}^{m}\left[2[n]_{q}+2-(1-\alpha)|b|\right]} Y_{n} \bar{z}^{n} \\
& =z-\sum_{n=2}^{\infty}\left[X_{n}\left(h_{n}(z)-z\right)\right]+\sum_{n=1}^{\infty}\left[Y_{n}\left(g_{m_{n}}(z)-z\right)\right] \\
& =\sum_{n=1}^{\infty}\left(X_{n} h_{n}+Y_{n} g_{m_{n}}\right) .
\end{aligned}
$$

Now from Theorem 2.2, we can deduce that $0 \leq X_{n} \leq 1,(n \geq 2)$ and $0 \leq Y_{n} \leq 1,(n \geq 1)$. Therefore $X_{1}=1-\sum_{n=2}^{\infty} X_{n}-\sum_{n=1}^{\infty} Y_{n} \geq 0$. Thus $\sum_{n=1}^{\infty}\left(X_{n} h_{n}+Y_{n} g_{m_{n}}\right)=f_{m}(z)$ as required in the theorem.

Finally, we determine the distortion theorem for the family $\overline{\mathcal{H}} S_{q}^{m}(b, \gamma, \alpha)$. 
Theorem 2.5. Let $f_{m} \in \overline{\mathcal{H}} \mathcal{S}_{q}^{m}(b, \gamma, \alpha)$ where $|z|=r<1$. Then

$$
\left|f_{m}(z)\right| \leq\left(1+b_{1}\right) r+\left(\frac{(1-\alpha)|b|}{[2]_{q}^{m}\left[2[2]_{q}-2+(1-\alpha)|b|\right]}-\frac{4-(1-\alpha)|b|}{[2]_{q}^{m}\left[2[2]_{q}-2+(1-\alpha)|b|\right]}\left|b_{1}\right|\right) r^{2}
$$

and

$$
\left|f_{m}(z)\right| \geq\left(1-b_{1}\right) r-\left(\frac{(1-\alpha)|b|}{[2]_{q}^{m}\left[2[2]_{q}-2+(1-\alpha)|b|\right]}-\frac{4-(1-\alpha)|b|}{[2]_{q}^{m}\left[2[2]_{q}-2+(1-\alpha)|b|\right]}\left|b_{1}\right|\right) r^{2} .
$$

Proof. We will prove the right hand inequality. The proof for the left hand inequality will be similar and is omitted. Let $f_{m}(z) \in \overline{\mathcal{H}} \mathcal{S}_{q}^{m}(b, \gamma, \alpha)$. Upon taking the absolute value of $f_{m}$, we obtain

$$
\begin{aligned}
\left|f_{m}(z)\right| \leq & \left.\left(1+\left|b_{1}\right|\right) r+\sum_{n=2}^{\infty}\left[\left|a_{n}\right|+\left|b_{n}\right|\right][n]_{q}^{m}\right) r^{n} \\
\leq & \left(1+\left|b_{1}\right|\right) r+r^{2} \sum_{n=2}^{\infty}\left(\left|a_{n}\right|+\left|b_{n}\right|\right)[n]_{q}^{m} \\
= & \left(1+\left|b_{1}\right|\right) r+\frac{(1-\alpha)|b| r^{2}}{[2]_{q}^{m}\left[2[n]_{q}-2+(1-\alpha)|b|\right]} \\
& \quad \times \sum_{n=2}^{\infty}[2]_{q}^{m}\left(\frac{2[2]_{q}-2+(1-\alpha)|b|}{(1-\alpha)|b|}\left|a_{n}\right|+\frac{2[2]_{q}-2+(1-\alpha)|b|}{(1-\alpha)|b|}\left|b_{n}\right|\right) \\
\leq & \left(1+\left|b_{1}\right|\right) r+\frac{(1-\alpha)|b| r^{2}}{[2]_{q}^{m}\left[2[2]_{q}-2+(1-\alpha)|b|\right]} \\
& \quad \times \sum_{n=2}^{\infty}[n]_{q}^{m}\left(\frac{2[n]_{q}-2+(1-\alpha)|b|}{(1-\alpha)|b|}\left|a_{n}\right|+\frac{2[n]_{q}+2-(1-\alpha)|b|}{(1-\alpha)|b|}\left|b_{n}\right|\right) \\
\leq & \left(1+\left|b_{1}\right|\right) r+\frac{(1-\alpha)|b|}{[2]_{q}^{m}\left[2[2]_{q}-2+(1-\alpha)|b|\right]}\left(1-\frac{[4-(1-\alpha)|b|]}{(1-\alpha)|b|}\left|b_{1}\right|\right) r^{2} \\
\leq & \left(1+\left|b_{1}\right|\right) r+\left(\frac{(1-\alpha)|b|}{[2]_{q}^{m}\left[2[2]_{q}-2+(1-\alpha)|b|\right]}-\frac{4-(1-\alpha)|b|}{[2]_{q}^{m}\left[2[2]_{q}-2+(1-\alpha)|b|\right]}\left|b_{1}\right|\right) r^{2} .
\end{aligned}
$$

The result is sharp for

$$
f(z)=z+\left|b_{1}\right| \bar{z}+\left(\frac{(1-\alpha)|b|}{[2]_{q}^{m}\left[2[2]_{q}-2+(1-\alpha)|b|\right]}-\frac{4-(1-\alpha)|b|}{[2]_{q}^{m}\left[2[2]_{q}-2+(1-\alpha)|b|\right]}\left|b_{1}\right|\right) \bar{z}^{2},
$$

where $\left|b_{1}\right| \leq \frac{(1-\alpha)|b|}{4-(1-\alpha)|b|}$.

\section{References}

[1] A. Aral, V. Gupta and R.P. Agarwal, Applications of q-calculus in operator theory, Springer, New York, 2013.

[2] Y. Avci and E. Zlotkiewicz, On harmonic univalent mappings, Ann. Univ. Mariae Curie- Sklodowska Sect. A, 44, 1-7, 1990.

[3] T. Bulboaca, M.A. Nasr and G.F. Sălăgean, A generalization of some classes of starlike functions of complex order, Mathematica (Cluj), 34 (57), 113-118, 1992.

[4] J. Clunie and T. Sheil-Small, Harmonic univalent Functions, Ann. Acad. Aci. Fenn. Ser. A.I. Math. 9, 3-25, 1984.

[5] M. Govindaraj and S. Sivasubramanian, On a class of analytic functions related to conic domains involving q-calculus, Anal. Math. 43(3)(5), 475-487, 2017.

[6] S.A. Halim and A. Janteng, Harmonic functions starlike of complex order, Proc. Int. Symp. on New Development of Geometric function Theory and its Applications, 132-140, 2008. 
[7] F.H. Jackson, On q-functions and a certain difference operator, Trans. Roy. Soc. Edinburgh, 46, 253-281, 1908.

[8] J.M. Jahangiri, Coefficient bounds and univalence criteria for harmonic functions with negative coefficients, Ann. Univ. Mariae Curie-Sk lodowska Sect. A, 5 (2), $57-$ 66, 1998.

[9] J.M. Jahangiri, Harmonic functions starlike in the unit disc, J. Math. Anal. Appl. 235, 470-477, 1999.

[10] J.M. Jahangiri, Harmonic univalent functions defined by q-calculus operators, Inter. J. Math. Anal. Appl. 5 (2), 39-43, 2018.

[11] J.M. Jahangiri, G. Murugusundaramoorthy and K. Vijaya, Sălăgean-Type harmonic univalent functions, Southwest J. Pure Appl. Math. 2, 77-82, 2002.

[12] J.M. Jahangiri, G. Murugusundaramoorthy and K. Vijaya, Starlikeness of Rucheweyh type harmonic univalent functions, J. Indian Acad. Math. 26, 191-200, 2004.

[13] S. Kanas, and D. Răducanu, Some subclass of analytic functions related to conic domains, Math. Slovaca, 64 (5), 1183-1196, 2014.

[14] T. Rosy, B.A. Stephen, K.G. Subramanian and J.M. Jagangiri, Goodman-Rønning type harmonic univalent functions, Kyungpook Math. J. 41, 45-54, 2001.

[15] G.F. Sălăgean, Subclasses of univalent functions, Springers-Verlog 1013, 362-372, 1983.

[16] H. Silverman, Harmonic univalent functions with negative coefficients, J. Math. Anal. Appl. 220, 283-289, 1998. 